\title{
Airbreathing Propulsion System Analysis Using Multithreaded Parallel Processing
}

\author{
Richard Gregory Schunk ${ }^{1}$ and T. J. Chung ${ }^{2}$ \\ 'NASA/MSFC, Huntsville, Alabama \\ ${ }^{2}$ Department of Mechanical \& Aerospace Engineering \\ The University of Alabama in Huntsville
}

\begin{abstract}
SUMMARY
In this paper, parallel processing is used to analyze the mixing and combustion behavior of hypersonic flow. Preliminary work for a sonic transverse hydrogen jet injected from a slot into a Mach 4 airstream in a two-dimensional duct combustor has been completed [Moon and Chung, 1996]. Our aim is to extend this work to three-dimensional domain using multithreaded domain decomposition parallel processing based on the flowfielddependent variation theory [Schunk, Canabal, Heard, and Chung, 1999; Schunk and Chung, 1999].
\end{abstract}

Numerical simulations of chemically reacting flows are difficult because of the strong interactions between the turbulent hydrodynamic and chemical processes. The algorithm must provide an accurate representation of the flowfield, since unphysical flowfield calculations will lead to the faulty loss or creation of species mass fraction, or even premature ignition, which in turn alters the flowfield information. Another difficulty arises from the disparity in time scales between the flowfield and chemical reactions, which may require the use of finite rate chemistry. The situations are more complex when there is a disparity in length scales involved in turbulence. In order to cope with these complicated physical phenomena, it is our plan to utilize the flowfield-dependent variation theory mentioned above, facilitated by large eddy simulation. Undoubtedly, the proposed computation requires the most sophisticated computational strategies. The multithreaded domain decomposition parallel processing will be necessary in order to reduce both computational time and storage. Without special treatments involved in computer engineering, our attempt to analyze the airbreathing combustion appears to be difficult, if not impossible. We describe in detail the parallel processing strategy below.

Multi-threaded programming is utilized to take advantage of multiple computational elements on the host computer. Typically, a multi-threaded process will spawn multiple threads which are allocated by the operating system to the available computational elements (or processors) within the system. If more than one processor is available, the threads may execute in parallel resulting in a significant reduction in excution time. If more threads are spawned than available processors, the threads appear to execute concurrently as the operating system decides which threads execute while the others wait. One unique advantage of multi-threaded programming on shared memory multiprocessor systems is the ability to share global memory. This alleviates the need for data exchange or message passing between threads as all global memory allocated by the parent process is available to each thread. However, precautions must be taken to prevent deadlock or 
race conditions resulting from multiple threads trying to simultaneously write to the same data.

Threads are implemented by linking an application to a shared library and making calls to the routines within that library. Two popular implementations are widely used: the Pthreads library (and its derivatives) that are available on most Unix operating systems and the NTthreads library that is available under Windows NT. There are differences between the two implementations, but applications can be ported from one to the other with moderate ease and many of the basic functions are similar albeit with different names and syntax.

Domain decomposition methods can be used in conjunction with multi-threaded programming to create an efficient parallel application. The sub-domains resulting from the decomposition provide a convenient division of labor for the processing elements within the host computer. In this application, an Additive Schwarz domain decomposition method is utilized. The method is illustrated below (Figure 1) for a two dimensional square mesh that is decomposed into four sub-domains. The nodes belonging to each of the four sub-domains are denoted with geometric symbols while boundary nodes are identified with bold crosses. The desire is to solve for each node implicitly within a single sub-domain. For nodes on the edge of each sub-domain this is accomplished by treating the adjacent node in the neighboring sub-domain as a boundary. The overlapping of neighboring nodes between sub-domains is illustrated in Figure 2. Higher degrees of overlapping, which may improve convergence at the expense of computation time, are also used.

In a parallel application, load balancing between processors is critical to achieving optimum performance. Ideally, if a domain could be decomposed into regions requiring an identical amount of computation, it would be a simple matter to divide the problem between processing elements as shown in Figure 3 for four threads executing on an equal number of processors.

Unfortunately, in a "real world" application the domain may not be decomposed such that the computation for each processor is balanced, resulting in lost efficiency. If the execution time required for each sub-domain is not identical, the CPU's will become idle for portions of time as shown in Figure 4.

One approach to load balancing, as implemented in this application, is to decompose the domain into more sub-domains than available processors and use threads to perform the computations within each block. The finer granularity permits a more even distribution of work amongst the available processing elements as shown in Figure 5.

In this approach, the number of threads spawned is equal to the number of available processors with each thread marching through the available sub-domains (which preferably number at least two times the number of processors), solving one at a time in an "assembly-line" fashion. A stack is employed where each thread pops the next subdomain to be solved off of the top of the stack. Mutual exclusion locks are employed to protect the stack pointer in the event two or more threads access the stack simultaneously. Each thread remains busy until the number of sub-domains is exhausted. If the number of 
sub-domains is large enough, the degree of parallelism will be high although decomposing a problem into too many sub-domains may adversely affect convergence.

The above approach will be utilized for the analysis of hypersonic airbreathing combustion with hydrogen fuel. Without using the parallel processing, the analysis with Mach 4 free-stream velocity for the finite rate chemistry with 18 species has been carried out as shown in Figs. 6 through 8 [Moon and Chung, 1996]. In this study, the standard $\mathrm{K}-\varepsilon$ model was used. The proposed paper will utilize the large eddy simulation with high Mach numbers and high Reynolds numbers. It is our plan to report on the maximum ranges of Mach number and Reynolds number the proposed algorithm can accommodate. Our eventual goal in this paper is to determine the relationship between mixing and combustion efficiency. Length scales and time scales involved in turbulence and combustion will be thoroughly investigated.

\section{References}

Moon, S.Y. and Chung, T. J. [1996]: Numerical simulation of heat transfer in chemically reacting shock wave turbulent boundary layer interactions, Journal of Numerical Heat Transfer, Part A, 30, 1,55-72.

Schunk, R. G, Canabal, F., Heard, G.A., and Chung, T.J. [1999]: Unified CFD methods via flowfield-dependent variation theory. AIAA paper 99-3715.

Schunk, R. G. and Chung, T. J. [1999]: Parallelization of the flowfield-dependent variation schemes for solving the triple shock/boundary layer interaction problem, TFAWS 99 NASA/MSFC, Septemer 13-15, 1999. 


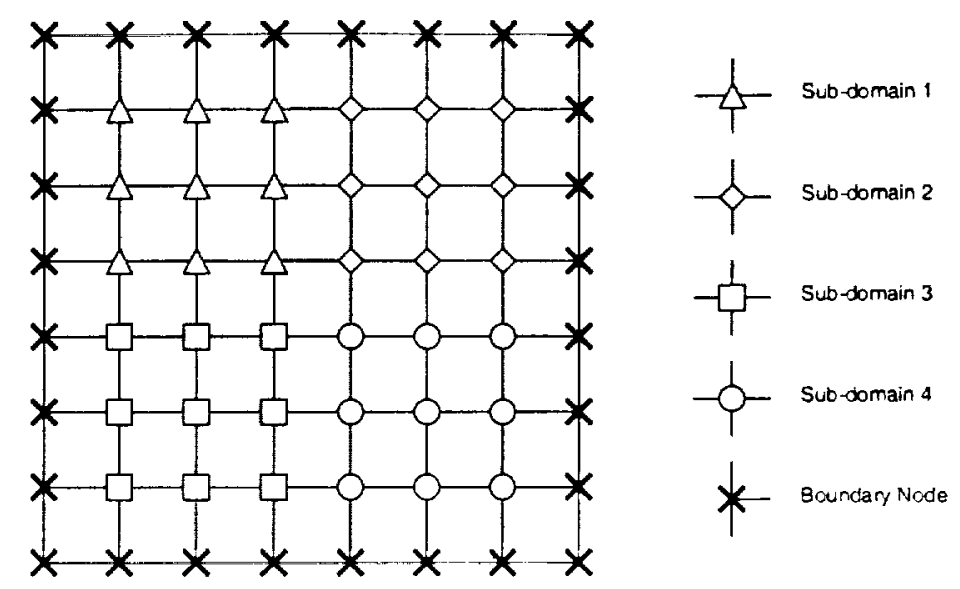

Figure 1: Multiple Subdomains

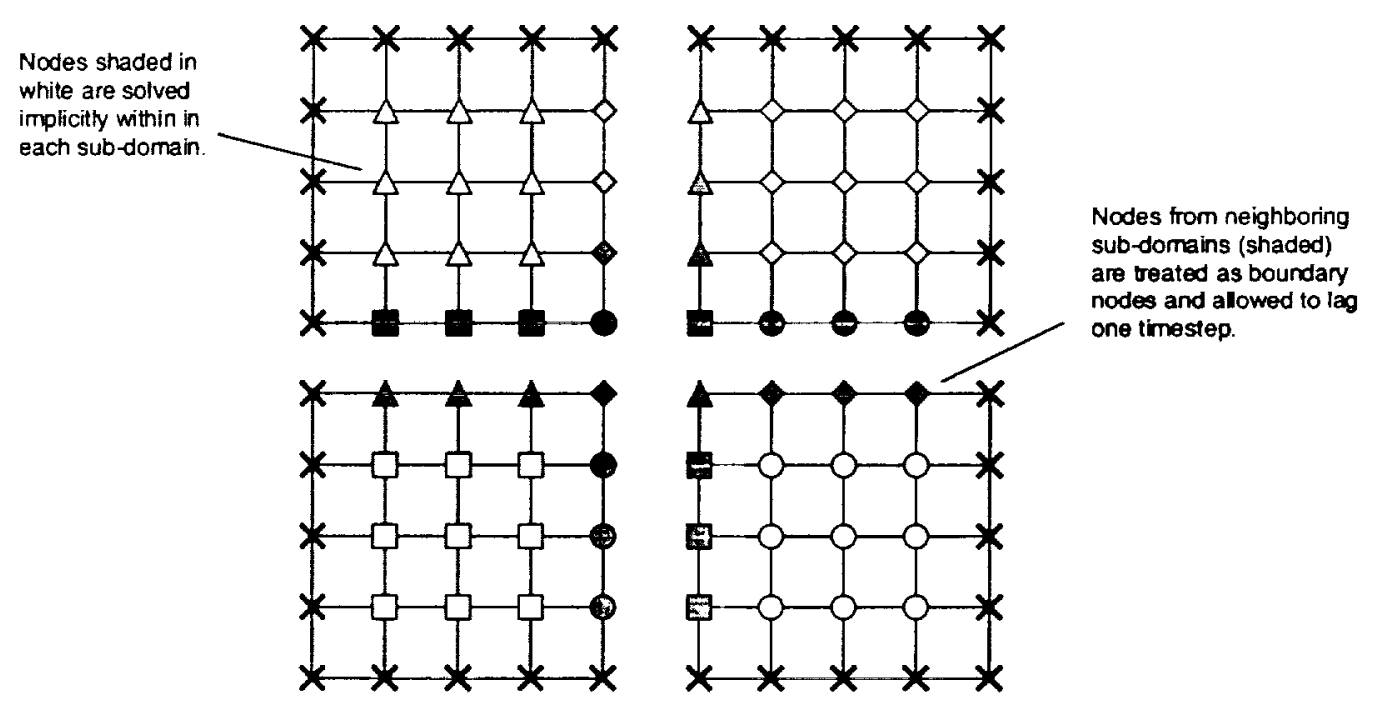

Figure 2: Domain Decomposition 


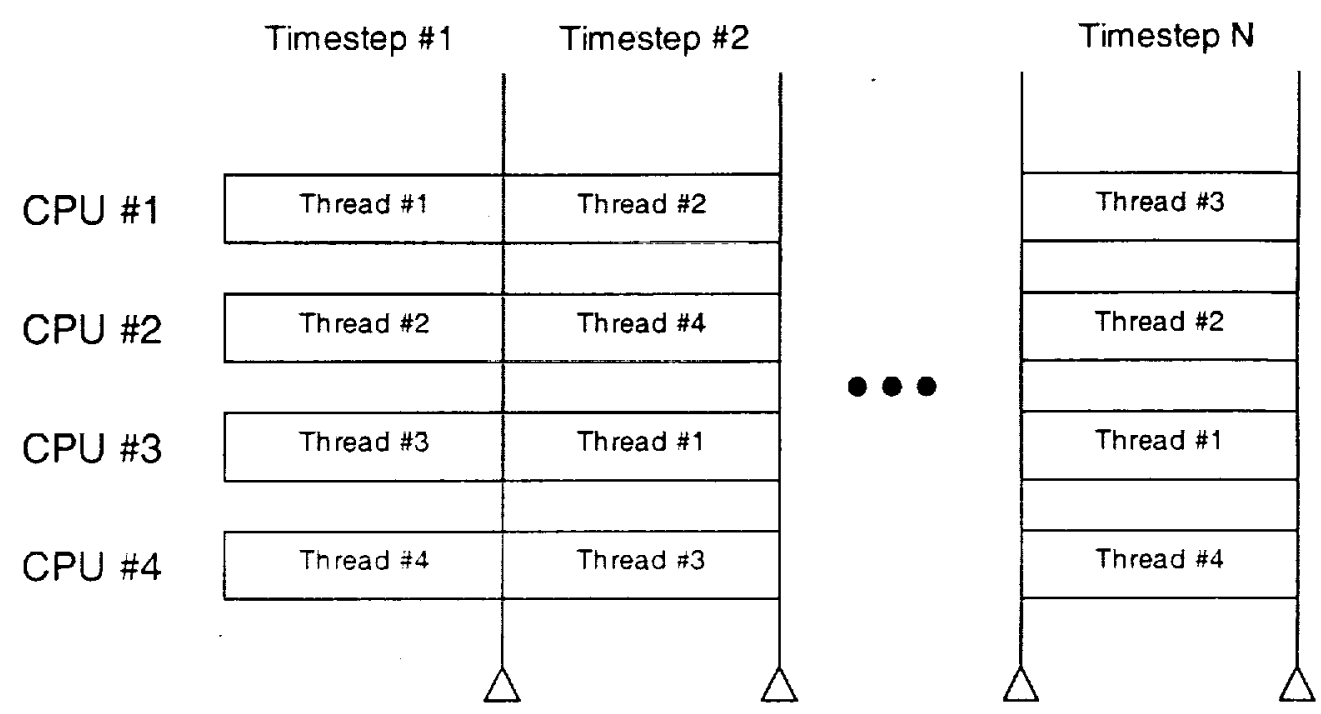

$\triangle$ End of Timestep Synchronization

Execution Time

Figure 3: Ideal Load Balancing

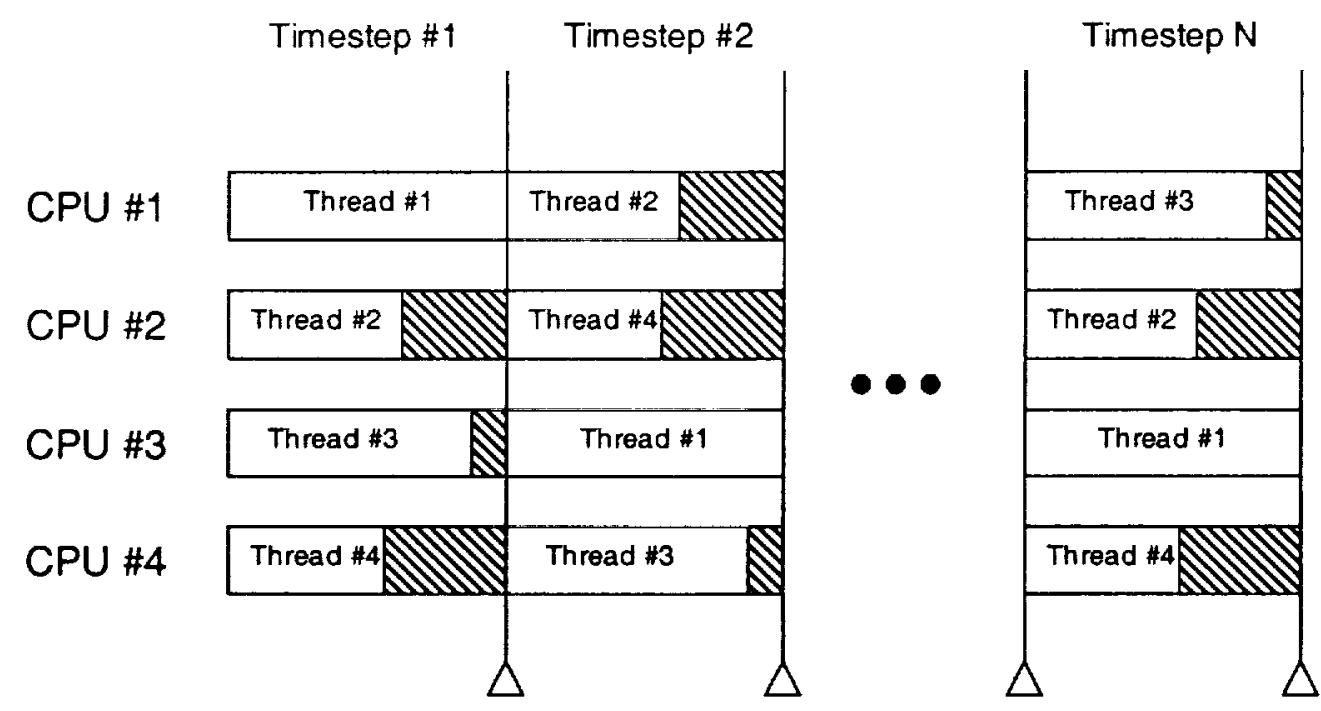

$\triangle \quad \begin{array}{r}\text { End of Timestep } \\ \text { Synchronization }\end{array}$

CPU Idle

Execution Time

Figure 4: "Real World" Load Balancing 


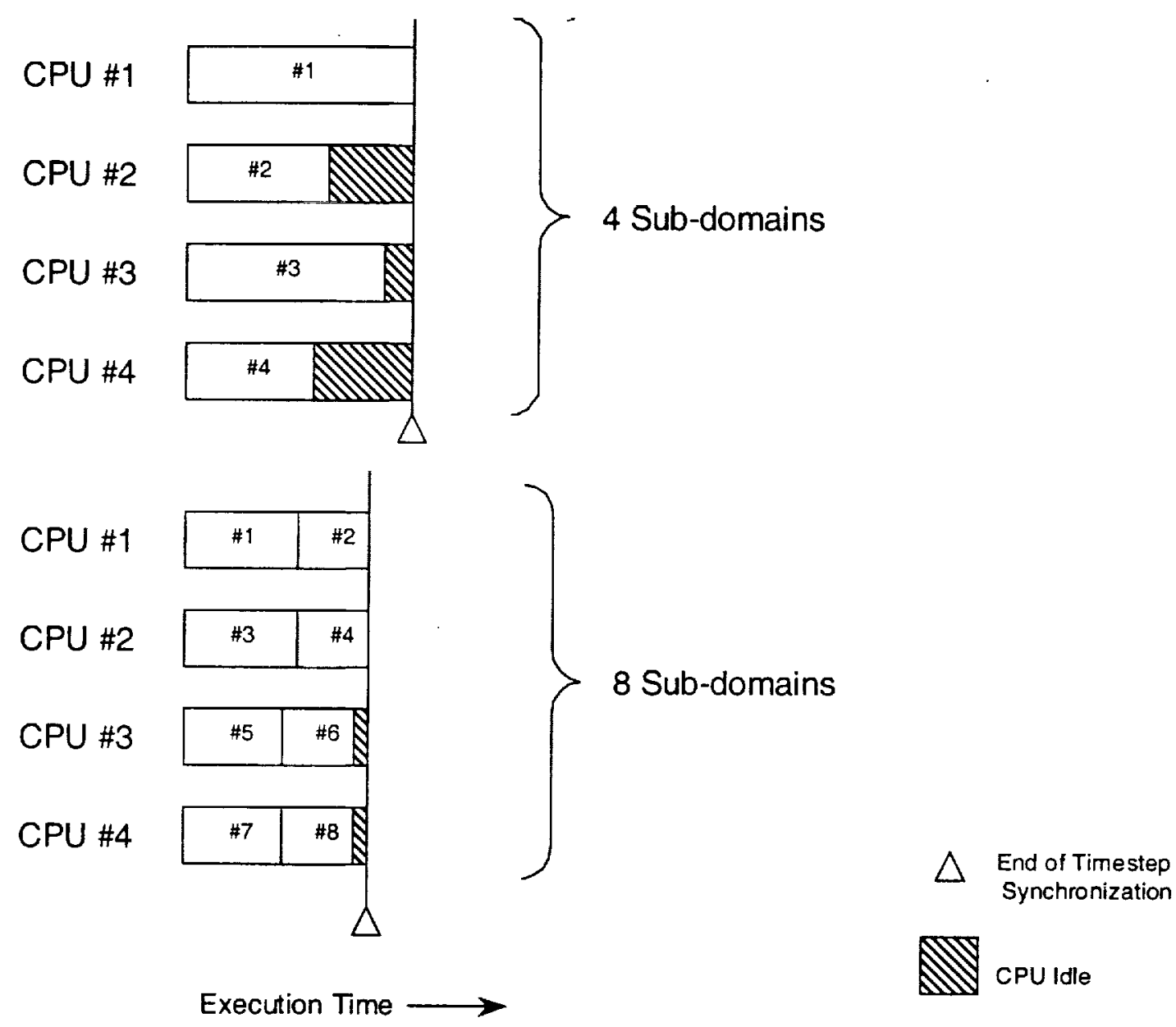

Figure 5: Domain Decomposition Improves Parallelism 


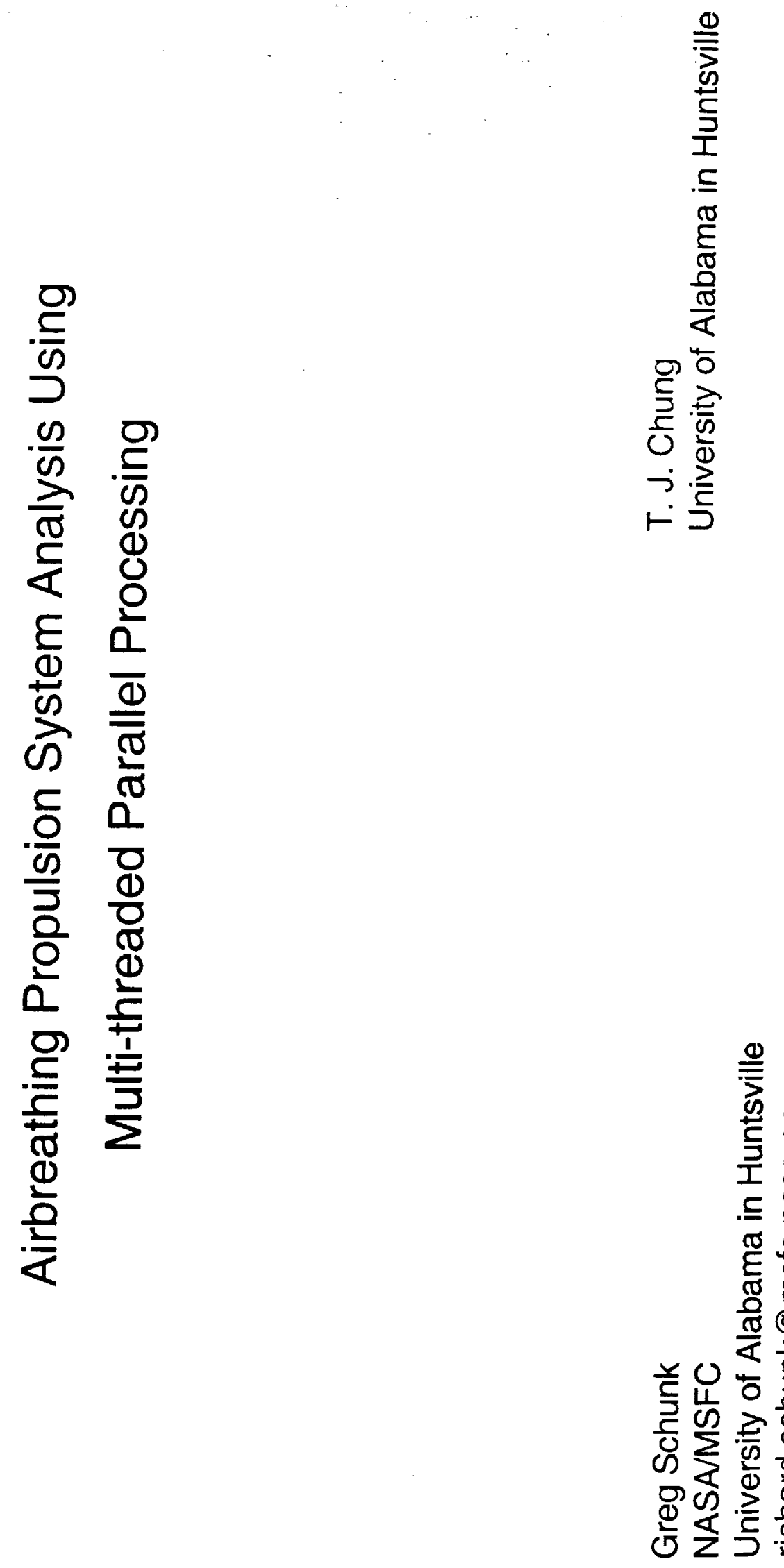




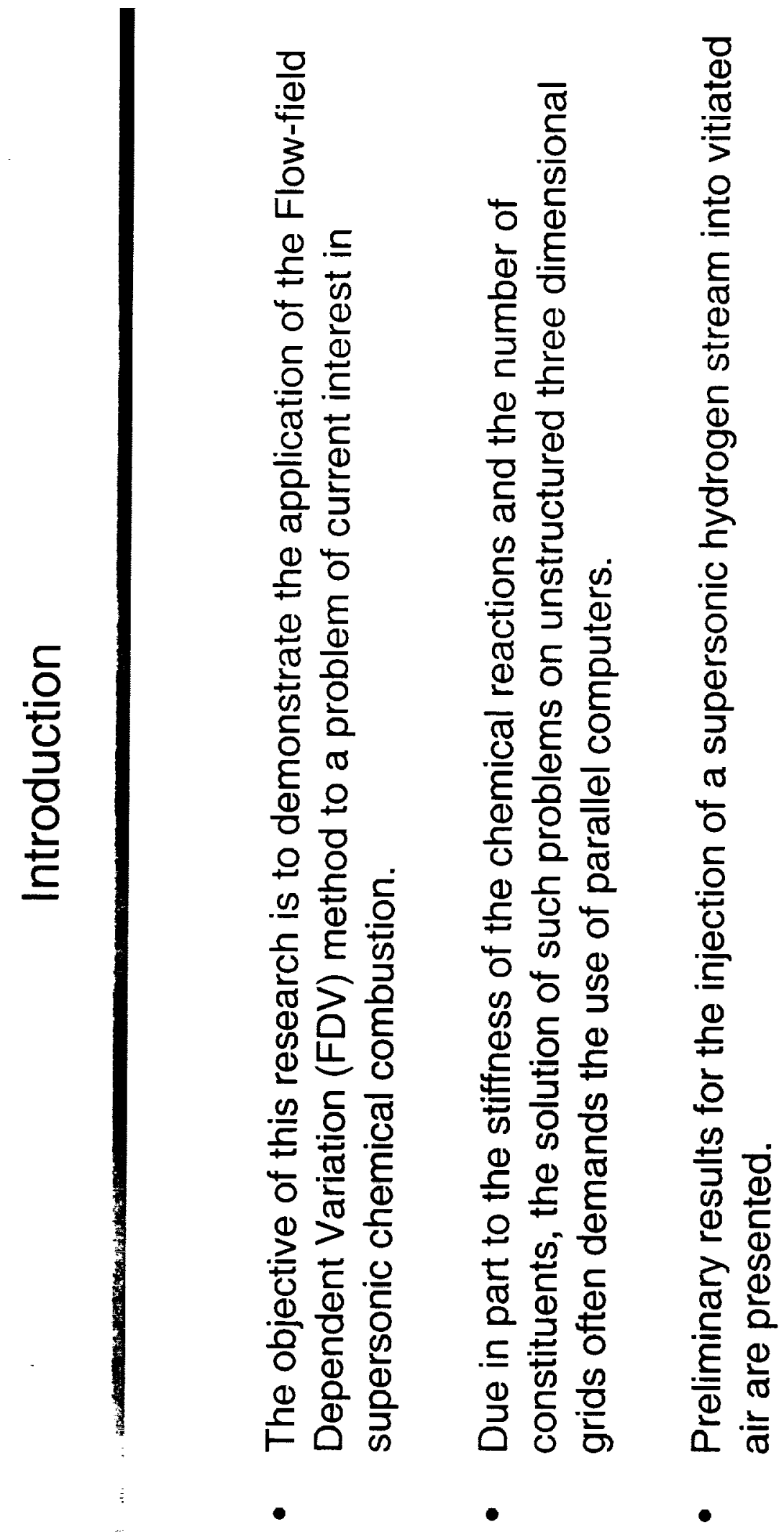




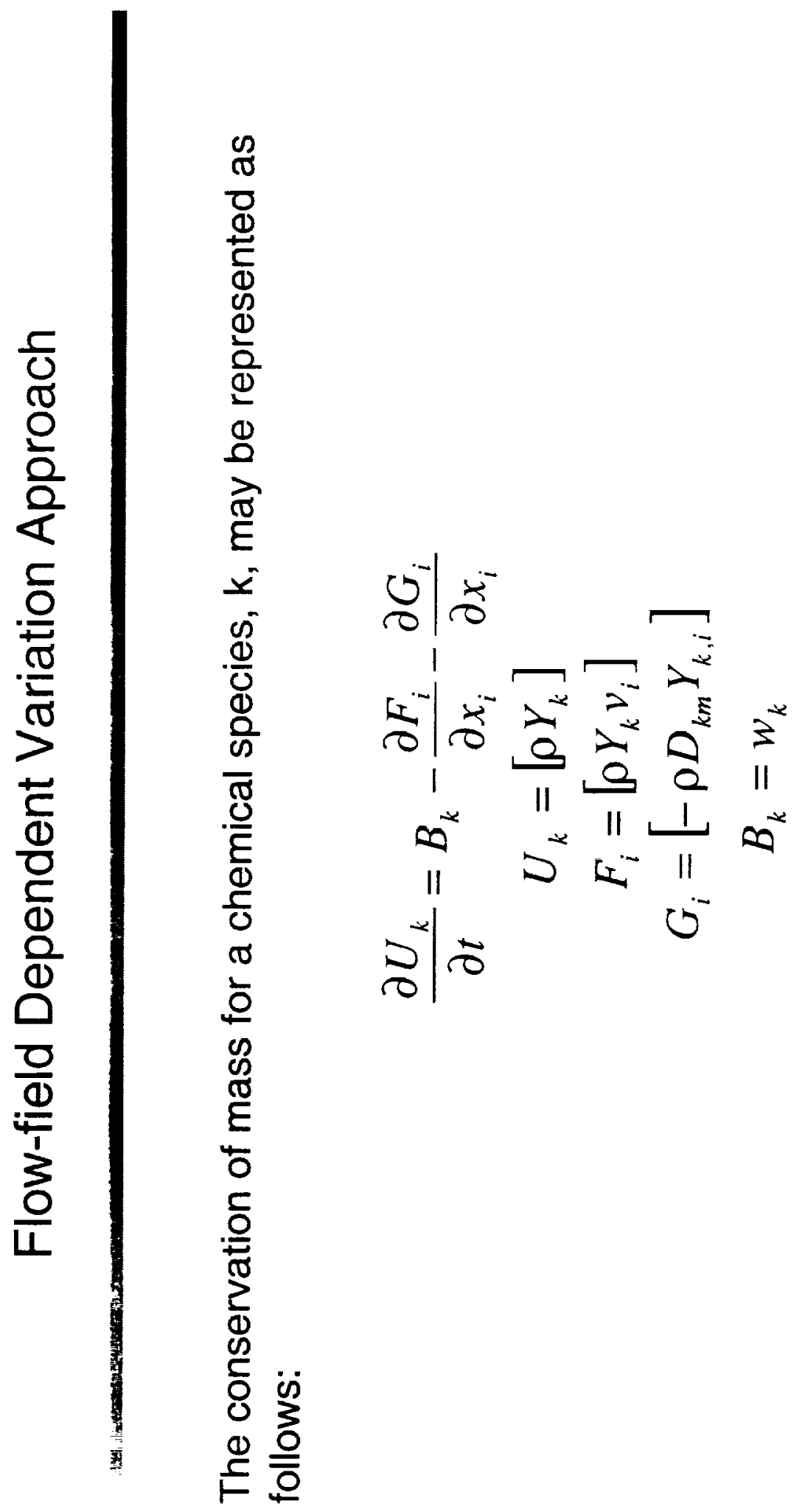

$\stackrel{\Phi}{\nsubseteq}$

요

$\circlearrowleft 5$

可

$0 \cong$

$\geqq \bar{\pi}$

일

$\geq$

인

틍

으

4 क

두 $\frac{9}{8}$

응

क्ष के

$\leftarrow$

ஸ 등

뽈 을

( )

$\frac{\Phi}{0} \frac{5}{0}$

$\Phi$ o

क응

$0 \neq$

도 $\underline{0}$

c) $3^{x}$

के

은 즐

$>4$

(2)

造 


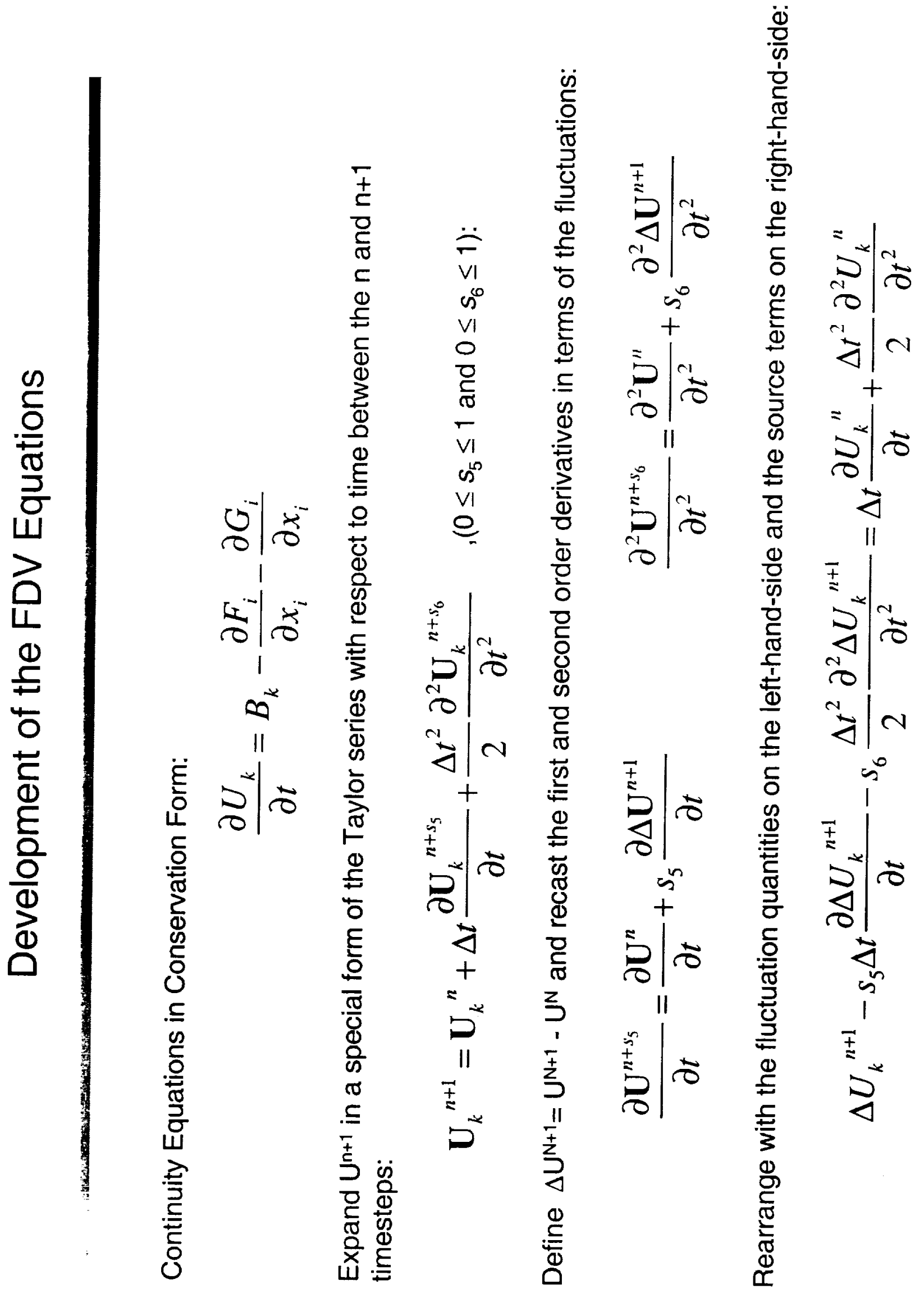



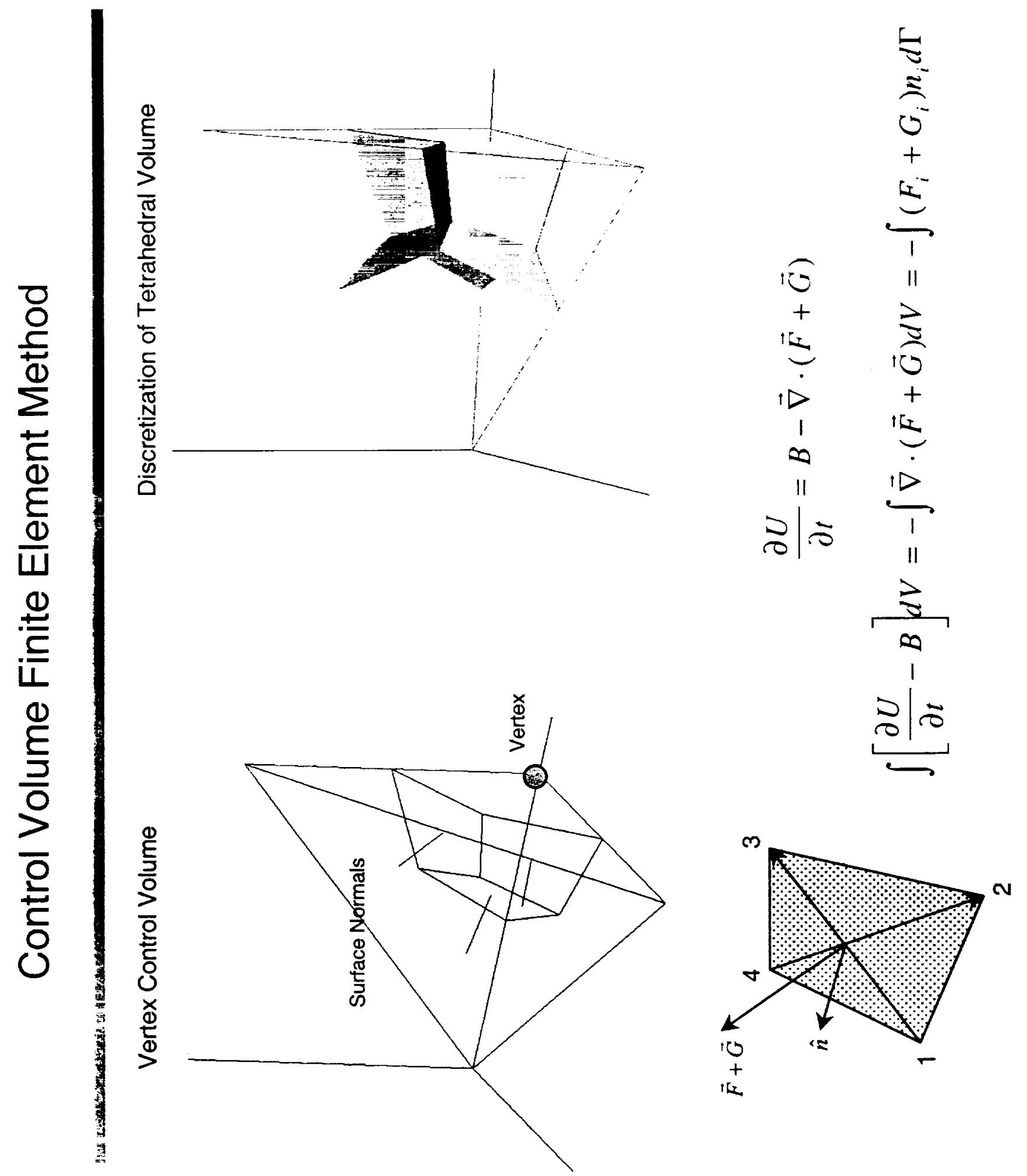

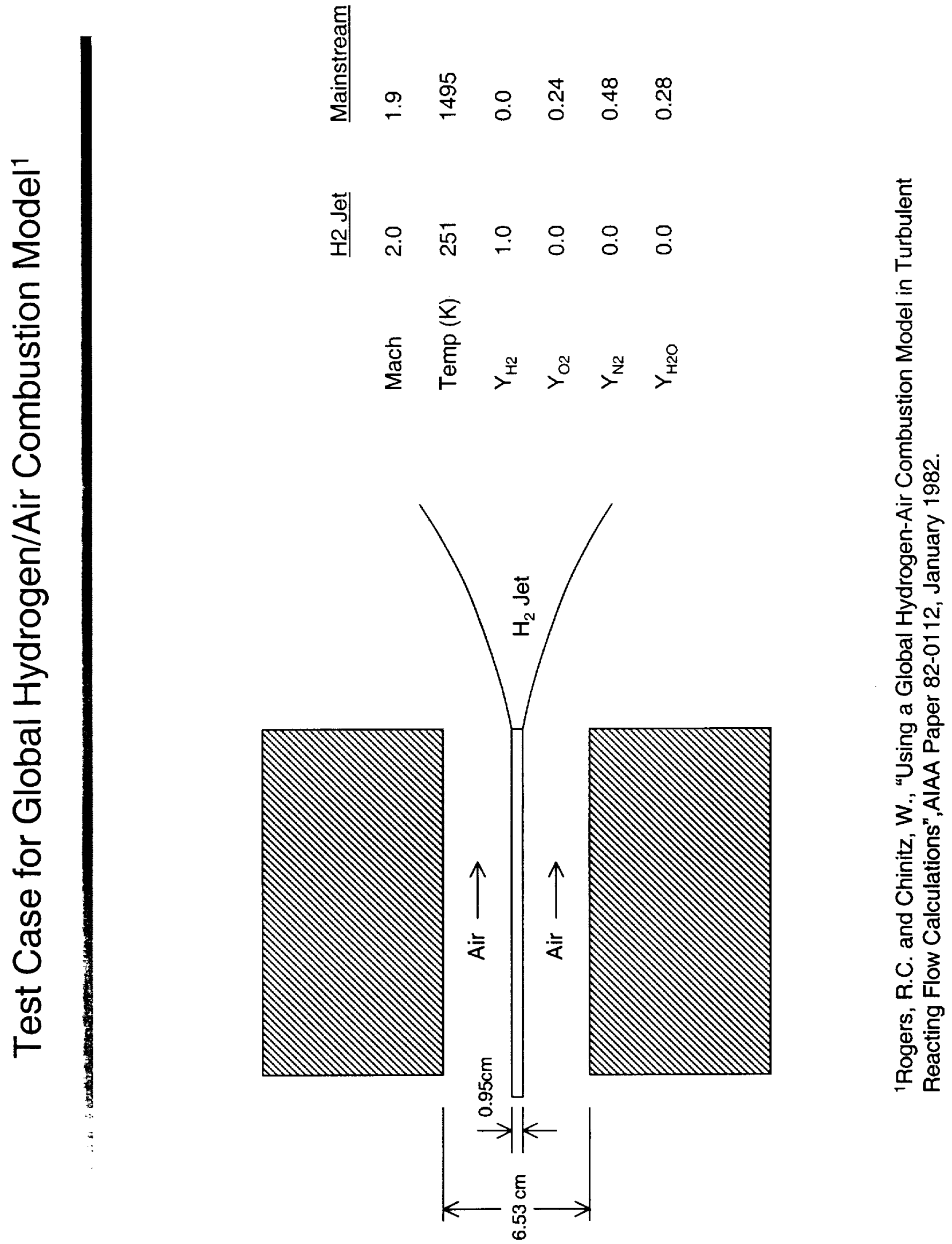


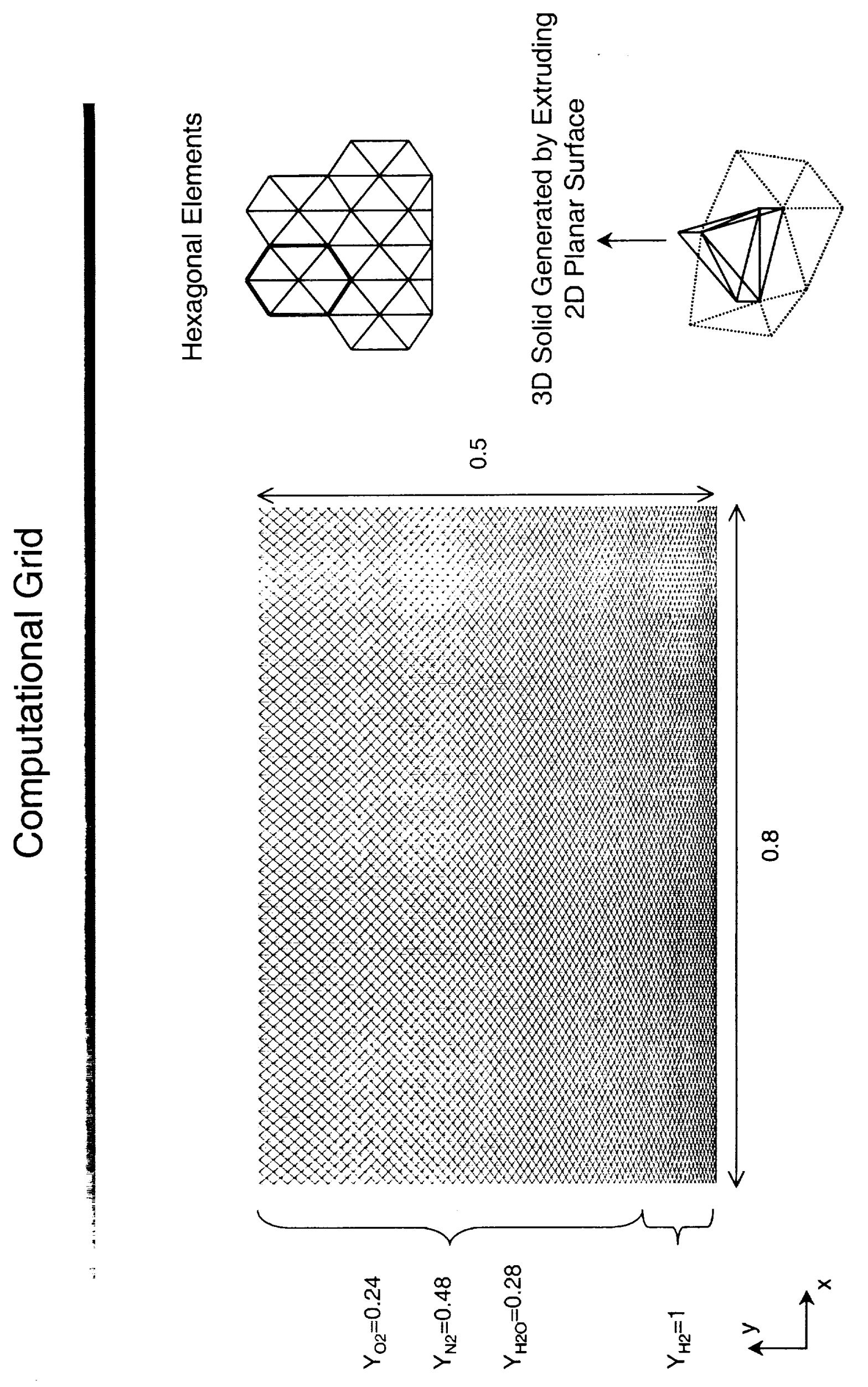



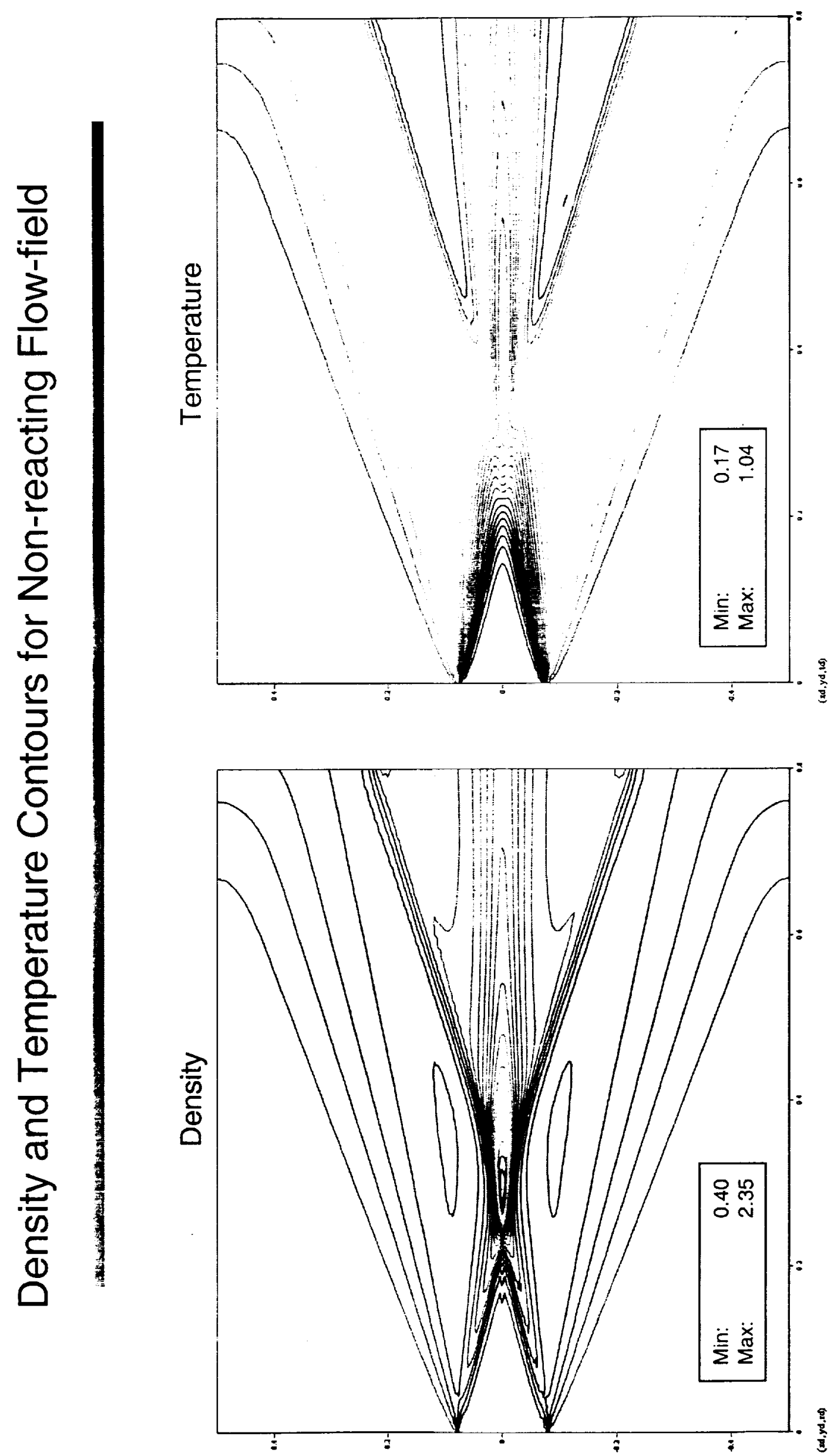


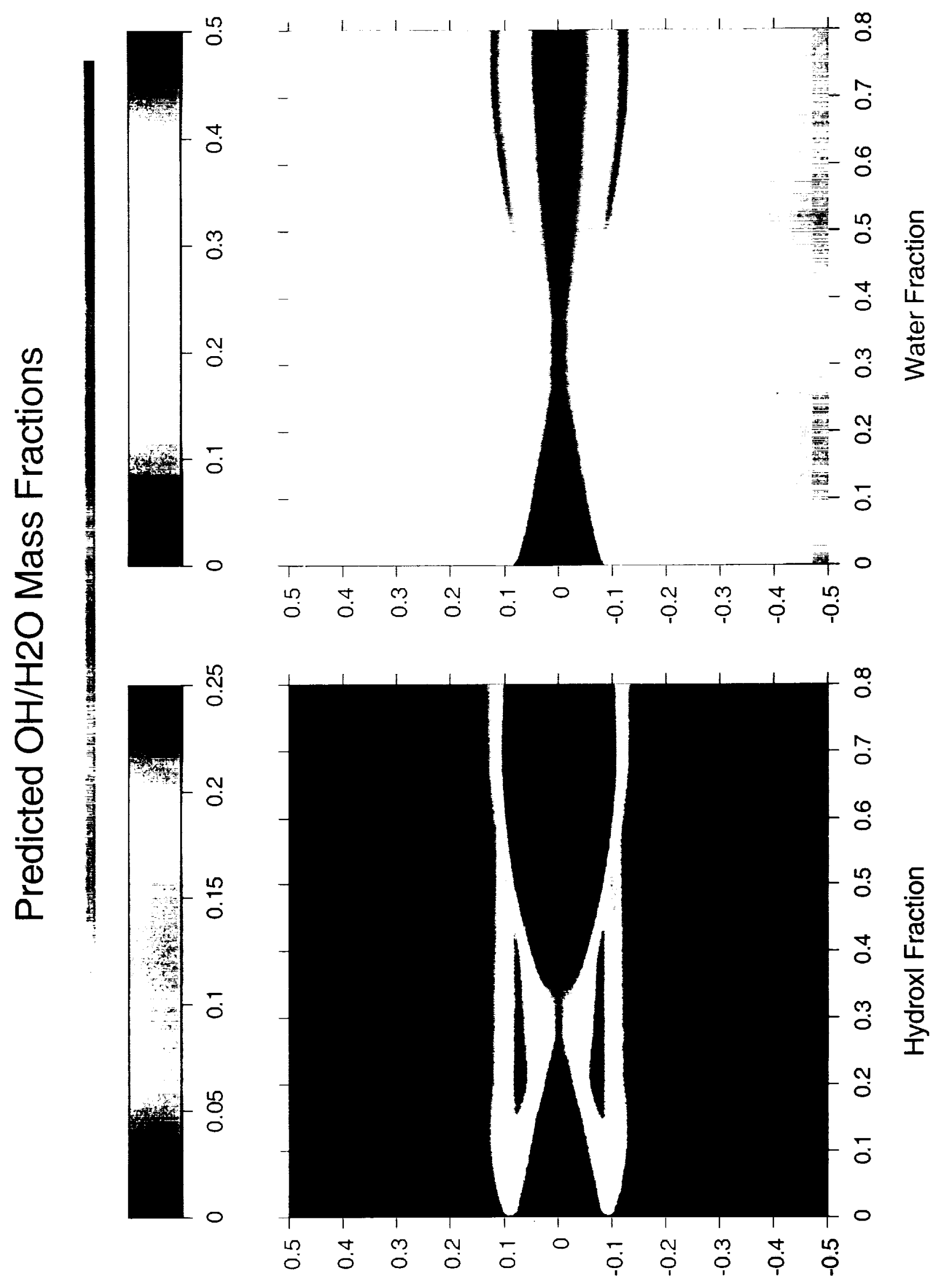




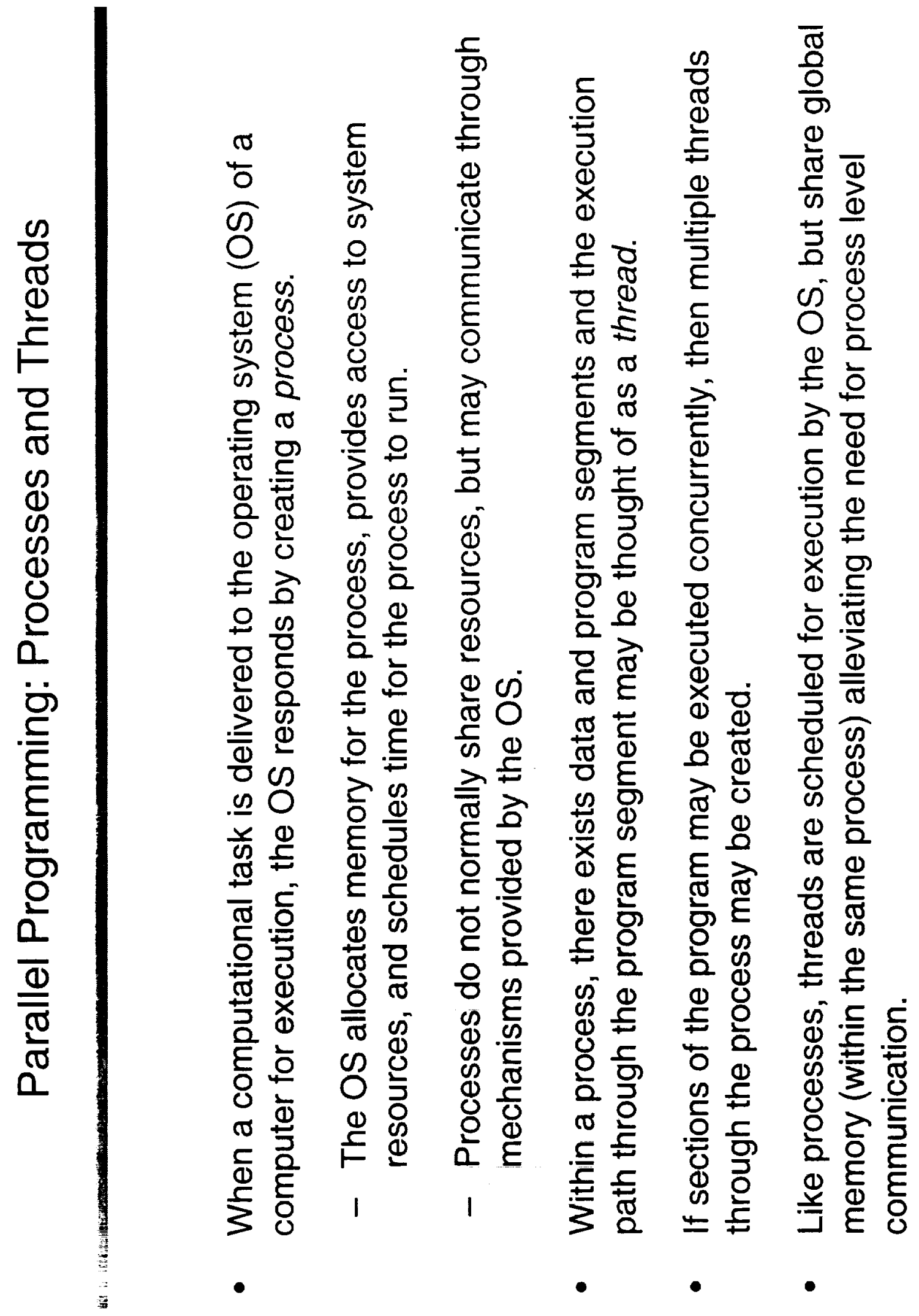




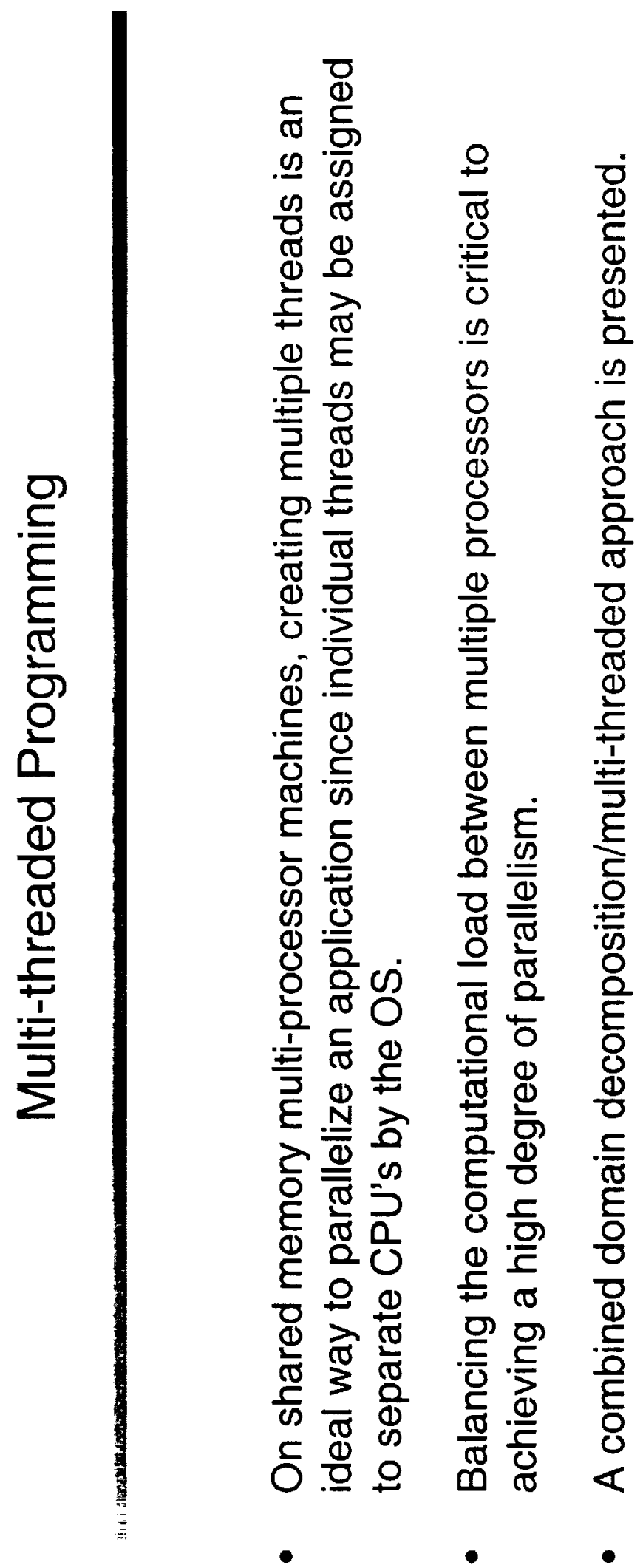




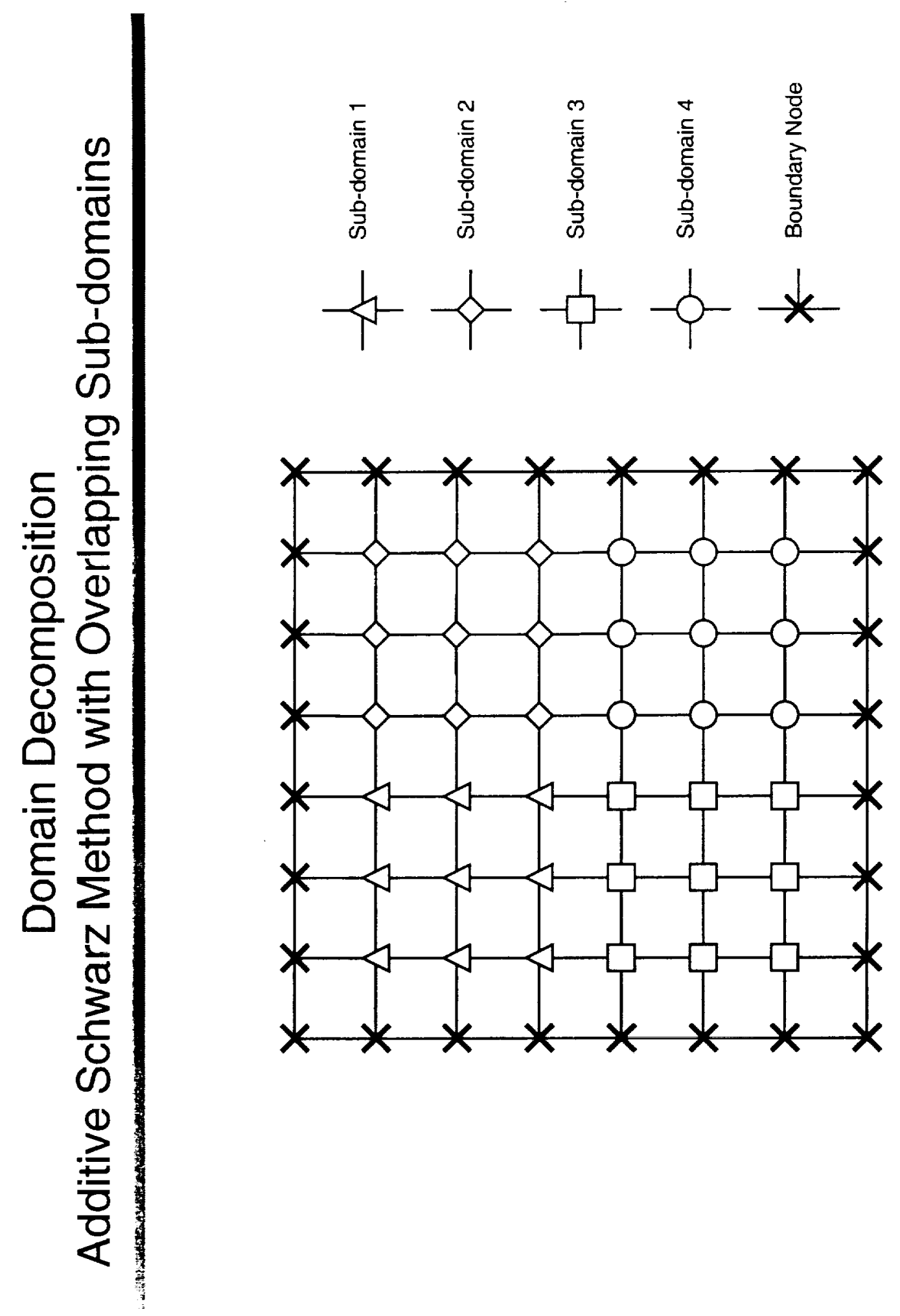




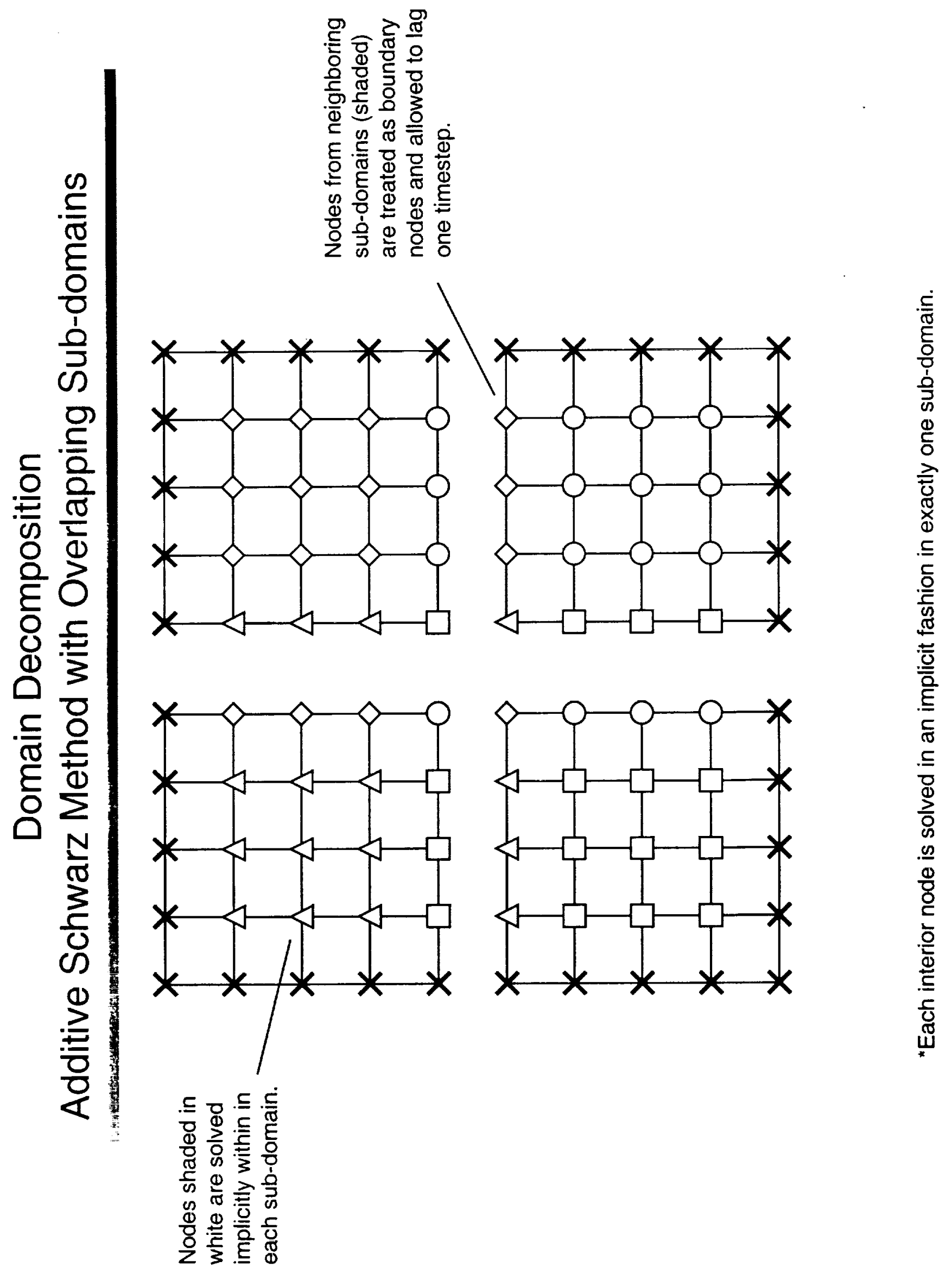




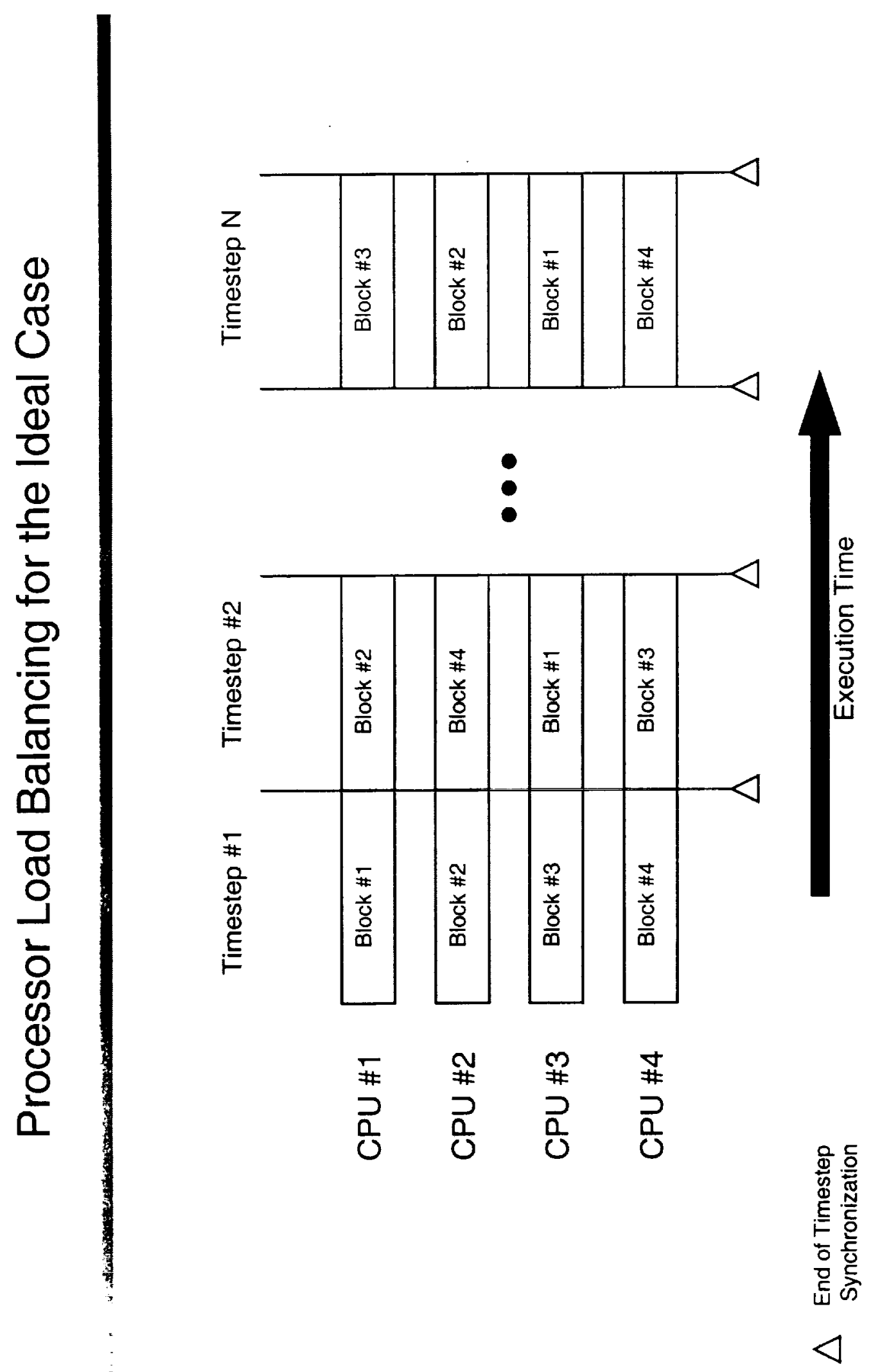









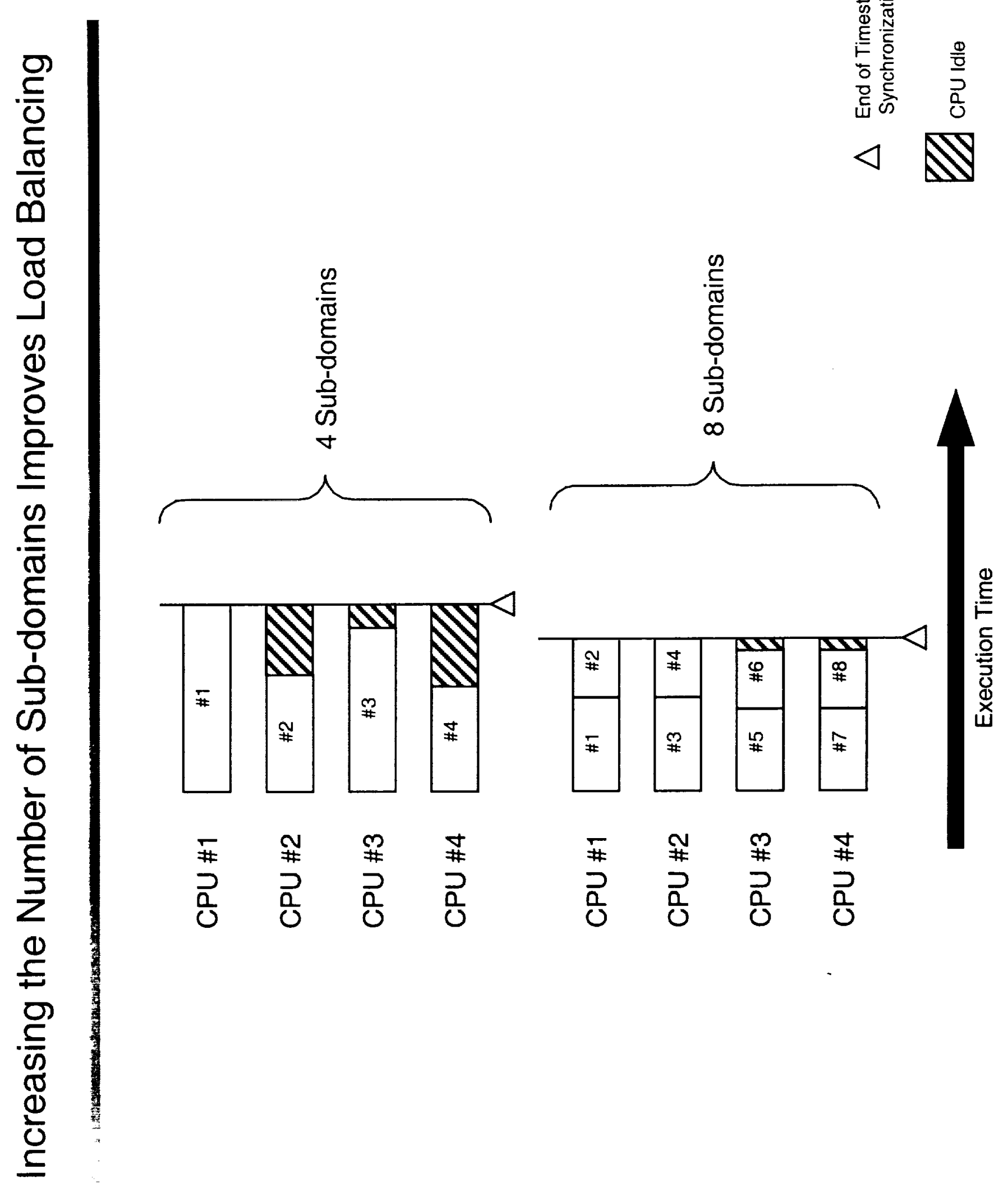




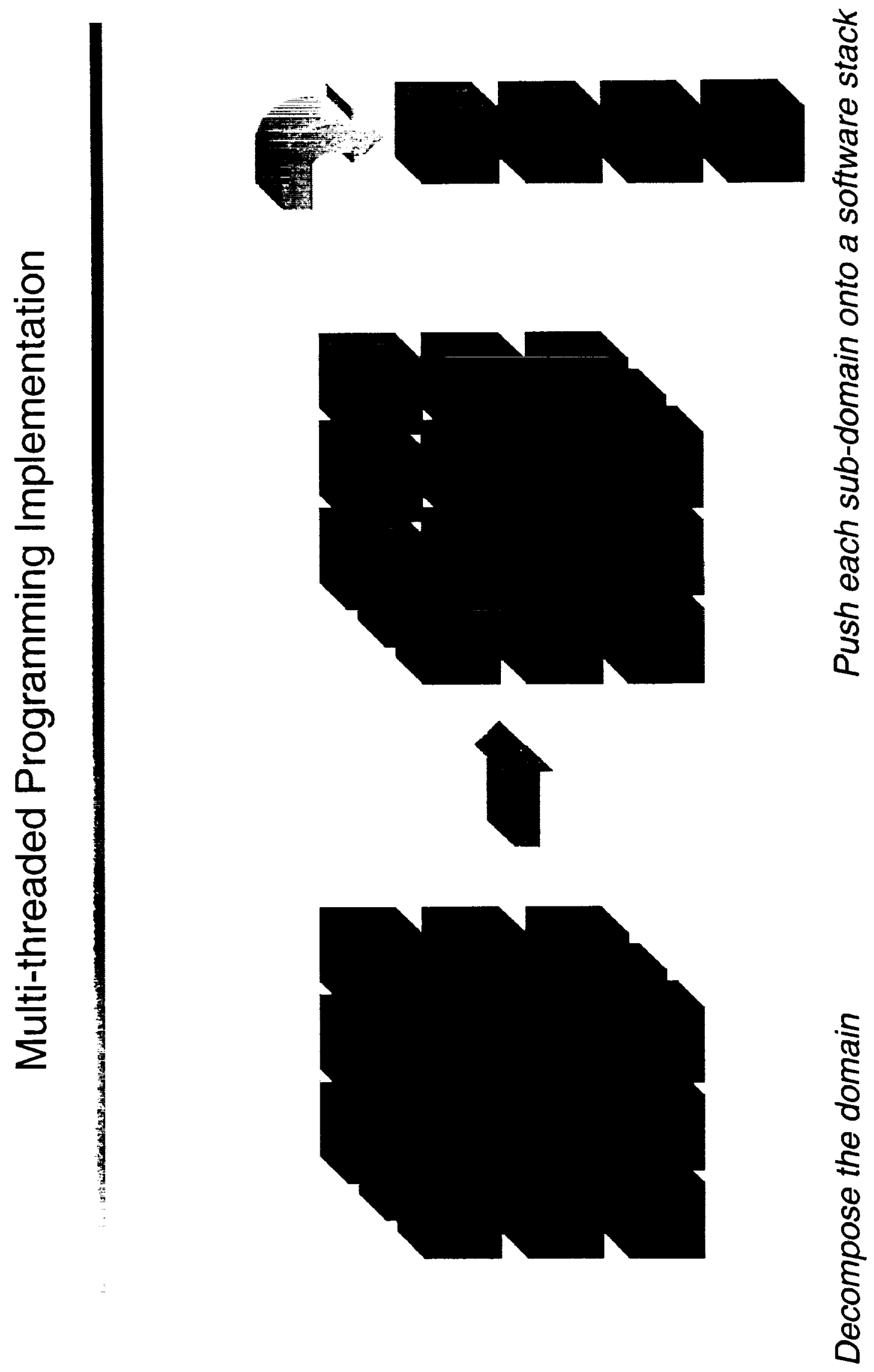




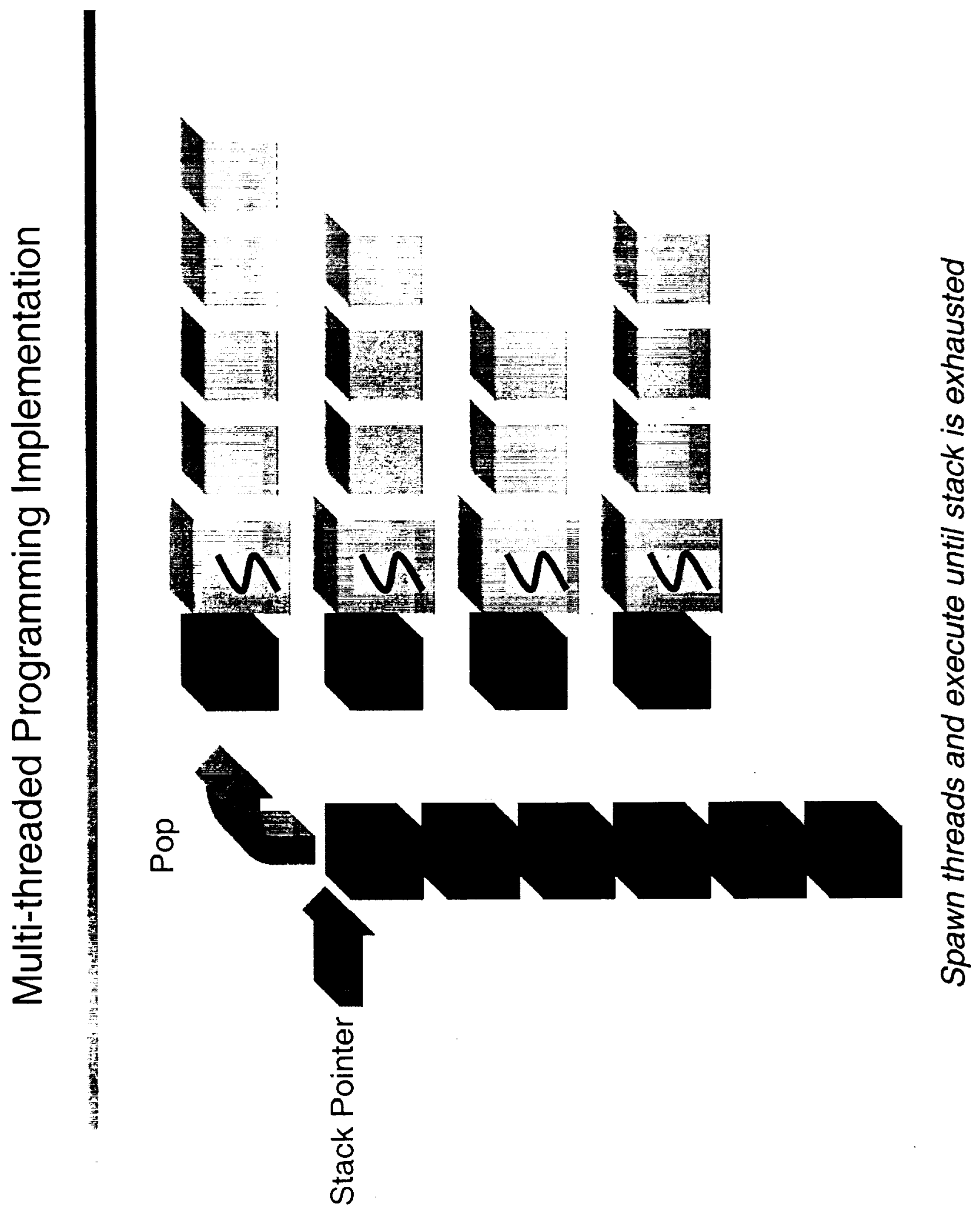




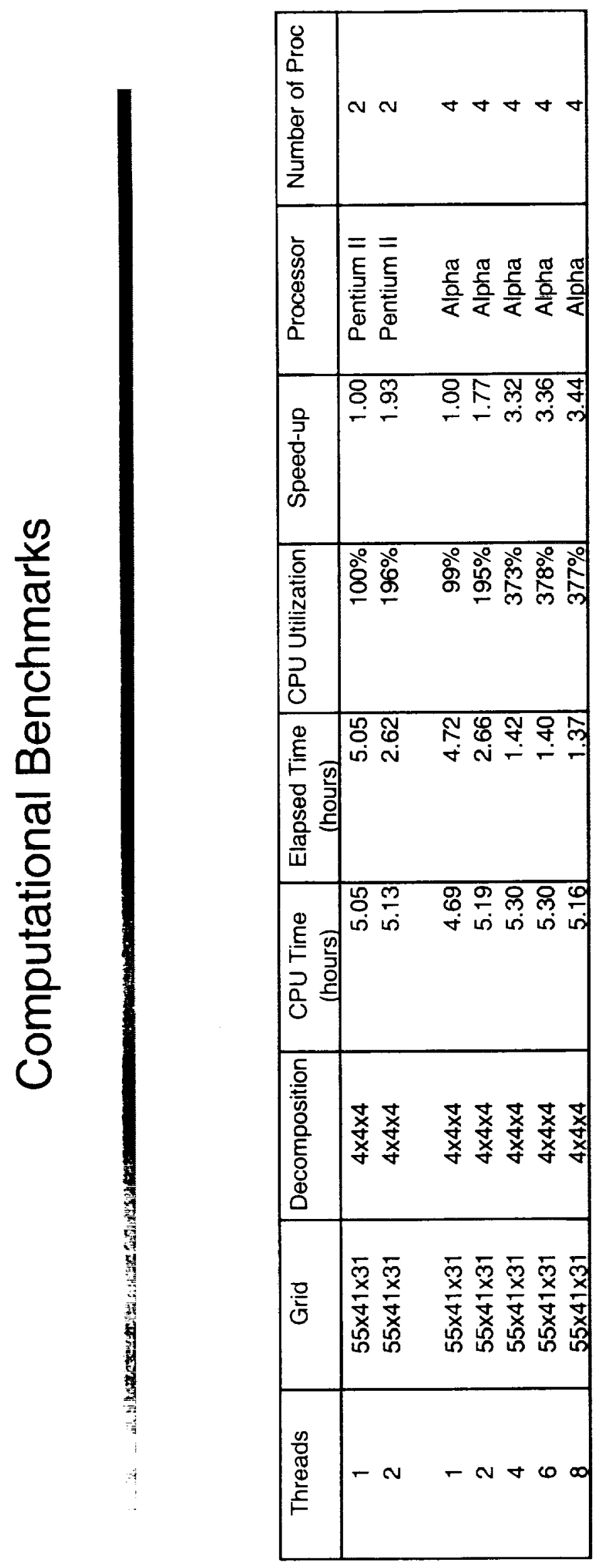




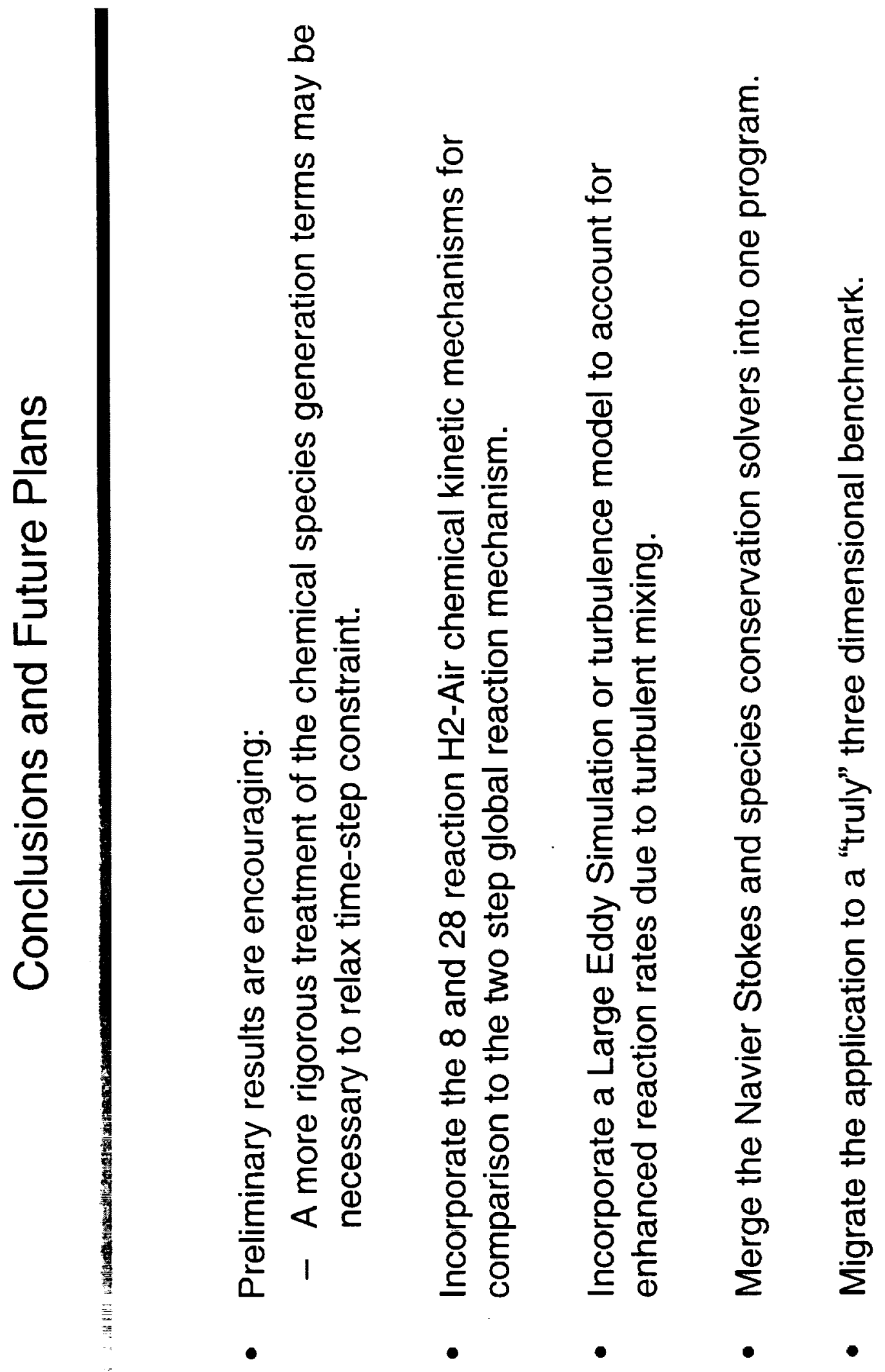

